\title{
Prediction of the Styrene Butadiene Rubber Performance by Emulsion Polymerization Using Backpropagation Neural Network
}

\author{
Yan-jiang Jin,, ${ }^{1,2}$ Ben-xian Shen, ${ }^{1}$ Ruo-fan Ren,, Lei Yang, ${ }^{2}$ Jun Sui,, and Ji-gang Zhao ${ }^{1}$ \\ ${ }^{1}$ State Key Laboratory of Chemical Engineering, East China University of Science and Technology, Shanghai 200237, China \\ ${ }^{2}$ Jilin Petrochemical Company, Petro China, Jilin 132021, China \\ Correspondence should be addressed to Ji-gang Zhao; zjg@ecust.edu.cn
}

Received 16 August 2012; Revised 29 November 2012; Accepted 1 December 2012

Academic Editor: Alireza Khataee

Copyright (C) 2013 Yan-jiang Jin et al. This is an open access article distributed under the Creative Commons Attribution License, which permits unrestricted use, distribution, and reproduction in any medium, provided the original work is properly cited.

\begin{abstract}
The effect of the amounts of initiator, emulsifier, and molecular weight regulator on the styrene butadiene rubber performance was investigated, based on the industrial original formula. It was found that the polymerization rate was increased with the increased dosage of initiator and emulsifier, and together with replenishing molecular weight regulator will make the Mooney viscosity of rubber meet the national standard when the conversion rate reaches $70 \%$. The backpropagation neural network was trained by the original formula and ameliorated formula on the basis of Levenberg-Marquardt algorithm, and the relative error between the simulation results and experimental data is less than $1 \%$. The good consistency shows that the BP neural network could predict the product performances in different formula conditions. It would pave the way for adjustment of the SBR formulation and prediction of the product performances.
\end{abstract}

\section{Introduction}

Scientific researches try to increase emulsion polymerization monomer conversion and reaction rate by improving the formula on the basis of styrene butadiene rubber's good performance for many years $[1,2]$. But because of too many formulas, the researchers face the advantages such as large amount of experiments, and the mutual influence of formulas when alter single additive amount or feeding mode and use orthogonal test. The experimental data cannot clearly reflect the influence of each factor in formula [3].

The scientific workers are looking for a model to simulate the relationship between the polymerized styrene butadiene rubber performance and formula, what can provide reference to adjust formula and predict the product properties [47]. The artificial neural network (ANN) is composed of a large number of neurons by communicating through the adjustable metric. It has many features, such as distributed information memory, massively parallel processing and the self-adapt learning function. The ANN is widely used in pattern recognition, information processing, intelligent control, system modeling and other fields. Especially the Error Backpropagation Training (BP network) can approximate every continuous function and has good ability for nonlinear mapping. The layer number, processing elements number, learning coefficient, and other parameters can be settled by case. The BP network plays a very important role in many application fields because of its flexibility $[8,9]$.

The effects of the amounts of initiator, emulsifier, and molecular weight regulator on the performances of styrene butadiene rubber by original formula were investigated. Based on the original formula and optimized formula, the input vector of BP network train was the proportion of original initiator, emulsion, molecular regulator, and the conversion rate; the target vector was the combining styrene content and Mooney viscosity of the product of butadiene styrene rubber. The network was trained by using the LevenbergMarquardt (L-M) model. The product properties of different formulas were simulated by the established model. 


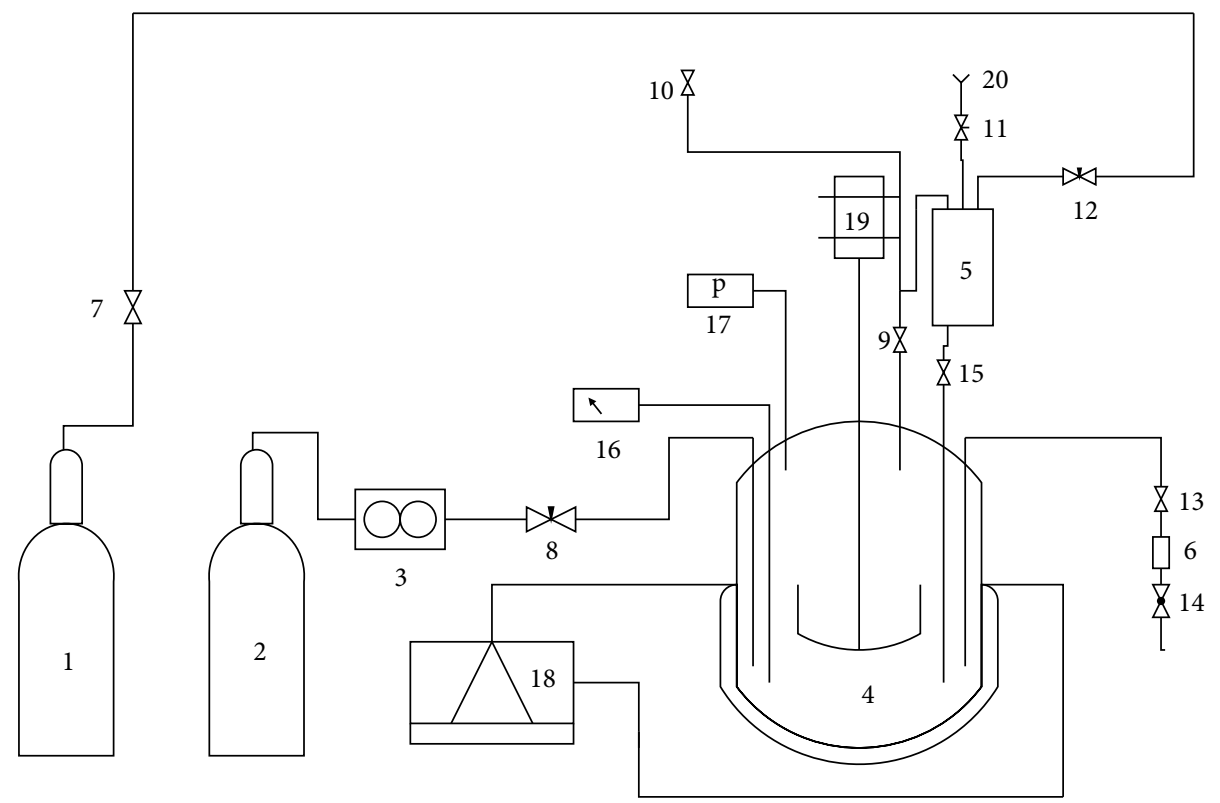

FIGURE 1: Process chart for emulsion polymerization. (1: Nitrogen, 2: butadiene, 3: mass flow meter, 4: autoclave, 5: feed tank, 6: sample buffer tank, 7 15: valves, 16: temperature indicator, 17: pressure indicator, 18: cold bath and the control device, 19: stirring motor, 20: feeding funnel).

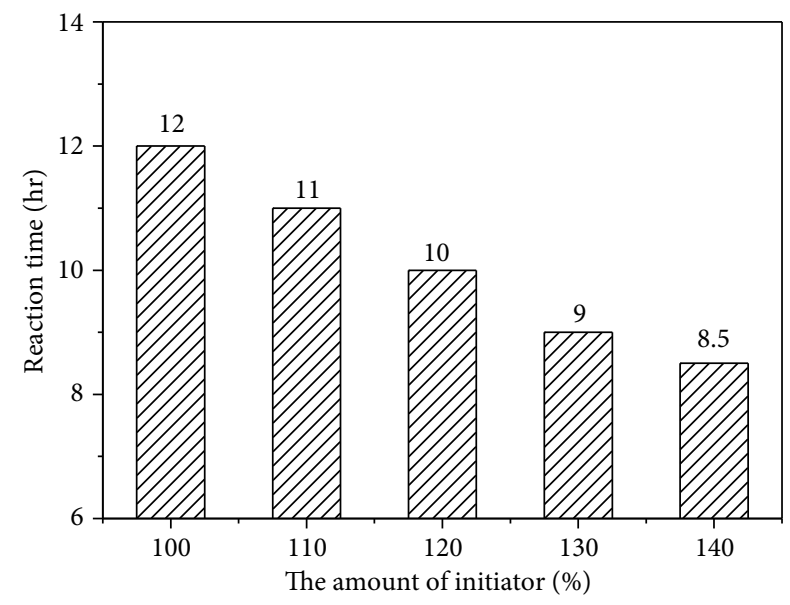

FIGURE 2: Effect amount of initiator on the emulsion polymerization rate.

\section{Experimental}

2.1. Materials. Butadiene (assay 99.9\%) was purchased from Weichuang gas company (Shanghai, China). Styrene (assay 99.9\%) was purchased from Lingfeng chemical agent company (Shanghai, China). K-ROSIN was the product of organic synthesis factory of Jilin petrochemical company. Tert-butyl hydroperoxide (assay 65\%) was delivered by Guoyao chemical agent company. Tert-dodecyl mercaptan (assay 99.9\%) was purchased by TCI shanghai company. Electrolyte solution (assay 10\%) was prepared in lab. Sodium hydrosulfite (assay 5.6\%) was produced by the organic synthesis factory of

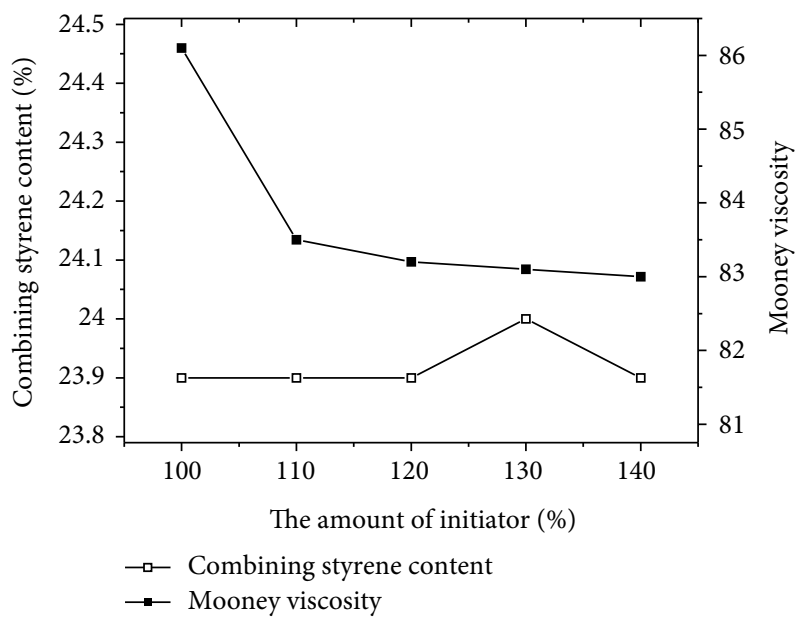

FIGURE 3: Effect amount of initiator on the combining styrene content and Mooney viscosity.

Jilin petrochemical company. Activator solution (assay $0.6 \%$ ) was prepared in lab.

2.2. Polymerization Reaction. The polymerization was reacted in $1.4 \mathrm{~L}$ reactor what is manufactured by Songling chemical company, Yantai, China, the polymerization flow chart as shown in Figure 1. The solutions were prepared firstly for accurate amount, and they were added to the reactor in quantization by order of sequence. The butadiene was calculated by mass flow meter and added into the reactor. The oxidizer, emulsifier, and molecular regulator were added by feed tank. The reaction was terminated by $1 \%$ 


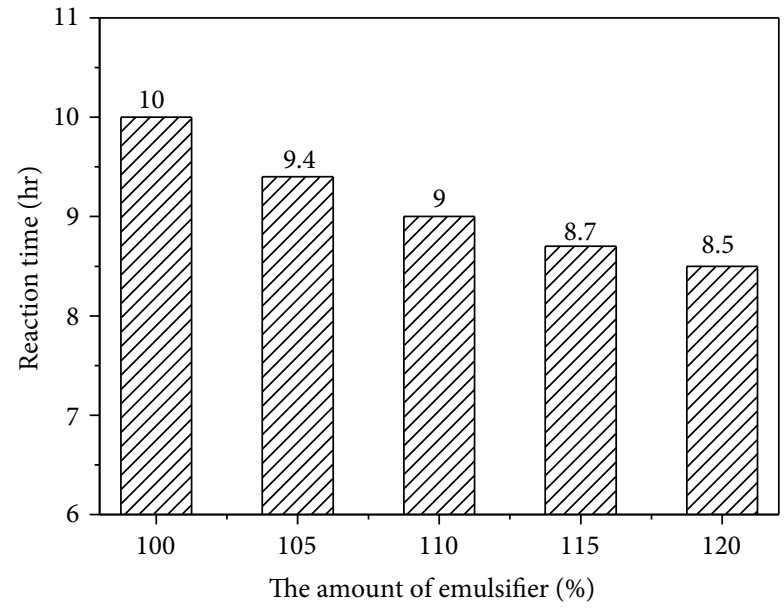

FIGURE 4: Effect amount of emulsifier on the emulsion polymerization rate.

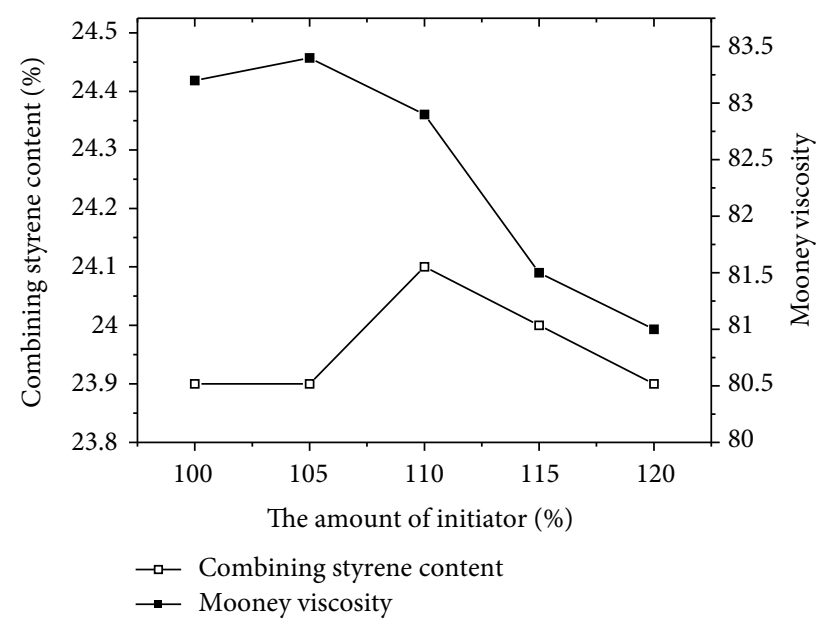

FIGURE 5: Effect amount of initiator on the combining styrene content and Mooney viscosity.

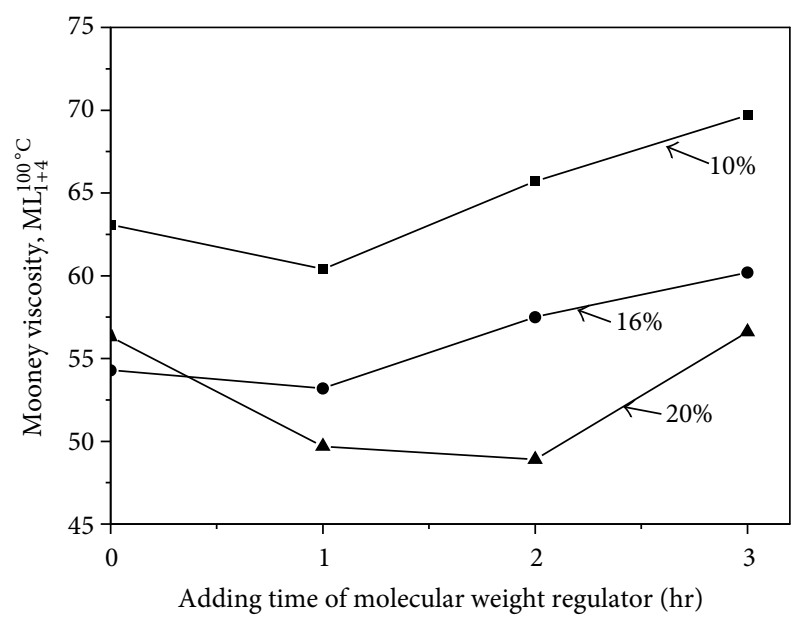

FIGURE 6: Effect of molecular weight regulator on the Mooney viscosity of rubber. hydroquinone solution after regular sampling. The samples were stored in ice bath for analysis.

2.3. Analysis and Characterization. The total solid content (TSC, m\%) was analyzed by Chinese SH/T 1154-1999 standard method. The $\mathrm{Ca}-\mathrm{H}_{2} \mathrm{SO}_{4}$ method was used for flocculation. The $\mathrm{pH}$ of emulsion was adjusted by $\mathrm{H}_{2} \mathrm{SO}_{4}$ to 3 , the emulsion flocculated by adding flocculant ( $1 \%$ styrenated phenols-methanol: $10 \%$, saline: $\left.3.4 \%, \mathrm{H}_{2} \mathrm{SO}_{4}=10: 2: 1\right)$ at $58 \sim 60^{\circ} \mathrm{C}$ under the agitation. If the solution became turbid, the $\mathrm{H}_{2} \mathrm{SO}_{4}$ can be added more.

The monomer conversion was evaluated by:

conversion $\%=2.95619 \times$ solid content $\times 100-5.616$.

The styrene content of rubber was determined by spectrophotometry according to Chinese GB/T 13646-92 (UV1900 Ultraviolet spectrophotometer from Yayan electrical company, Shanghai, China). The Mooney viscosity of rubber was determined according to Chinese GB/T 1232.1-2000 (JC2000G Mooney viscosity meter from Jingcheng instrument company, Jiangdu, China).

\section{Results and Discussion}

\subsection{Influence of Formula on Styrene-Butadiene Rubber}

3.1.1. Influence of Initiator Amount on Polymerization Rate. The influence of different initiator amount on polymerization rate was investigated. The reaction time of $70 \%$ monomer conversion was chosen to show the rate of polymerization for avoiding the impact of sampling. The results were shown in Figure 2. Figure 3 shows that the combining styrene content did not mainly change, but the Mooney viscosity decreased with the increasing of amount of initiator.

The results show that the polymerization rate can be increased by increasing the amount of initiator significantly. The polymerization time was reduced to 8.5 hours form 12 hours when the amount of initiator of original formula was increased by $40 \%$. Every $10 \%$ increase of initiator amount, the reductions of reaction time were different, the higher amount of initiator, the less reduction of reaction time. Because the rate of polymerization is depended on the amount of free radical. The more initiator, the more free radical, and the collision probability will increase.

3.1.2. Influence of Emulsion Amount on Polymerization Rate. The influence of different emulsion amount on polymerization rate was investigated. The reaction time of $70 \%$ monomer conversion was chosen to show the rate of polymerization for avoiding the impact of sampling. The results were shown in Figure 4. Figure 5 shows that that the combining styrene content maintain stability, and the Mooney viscosity decreased with the increasing of amount of initiator, but the Mooney viscosity value is also far away the Chinese national standard what is $46 \sim 58$.

The results show that the polymerization rate can be increased by increasing the amount of emulsion significantly. 


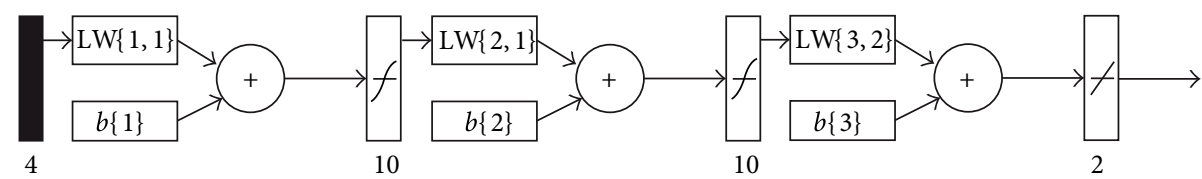

FIGURE 7: Structure of BP neural networks.

TABLE 1: SBR formulation conditions on the SBR products.

\begin{tabular}{|c|c|c|c|c|c|c|}
\hline \multirow[b]{2}{*}{ No. } & \multicolumn{3}{|c|}{ INPUT } & \multicolumn{3}{|c|}{ OUTPUT } \\
\hline & $\begin{array}{l}\text { Amount of } \\
\text { initiator, \% }\end{array}$ & $\begin{array}{c}\text { Amount of } \\
\text { Emulsifier, \% }\end{array}$ & $\begin{array}{l}\text { Amount of } \\
\text { molecular weight } \\
\text { regulator, } \%\end{array}$ & $\begin{array}{c}\text { Monomer } \\
\text { conversion, \% }\end{array}$ & $\begin{array}{l}\text { Combining } \\
\text { styrene } \\
\text { content, \% }\end{array}$ & Mooney viscosity, $\mathrm{ML}_{1+4}^{100^{\circ} \mathrm{C}}$ \\
\hline 1 & 100 & 100 & 100 & 61 & 22.9 & 50.4 \\
\hline 2 & 100 & 100 & 100 & 67 & 23.5 & 72.7 \\
\hline 3 & 100 & 100 & 100 & 71 & 23.9 & 86.1 \\
\hline 4 & 120 & 120 & 120 & 64.1 & 23.3 & 35.8 \\
\hline 5 & 120 & 120 & 120 & 70.5 & 24.1 & 49 \\
\hline 6 & 120 & 120 & 120 & 73.4 & 24.6 & 55.9 \\
\hline 7 & 120 & 120 & 120 & 75.2 & 25 & 66.9 \\
\hline 8 & 120 & 100 & 100 & 60 & 22.7 & 49.5 \\
\hline 9 & 120 & 100 & 100 & 70 & 23.9 & 83.2 \\
\hline 10 & 110 & 100 & 100 & 70 & 23.9 & 83.5 \\
\hline 11 & 130 & 100 & 100 & 70 & 24 & 83.1 \\
\hline 12 & 120 & 105 & 100 & 70 & 23.9 & 83.4 \\
\hline 13 & 120 & 110 & 100 & 70 & 24.1 & 82.9 \\
\hline 14 & 120 & 115 & 100 & 70 & 24 & 81.5 \\
\hline 15 & 120 & 120 & 100 & 70 & 23.9 & 81 \\
\hline 16 & 120 & 120 & 110 & 70 & 24.1 & 65.7 \\
\hline 17 & 120 & 120 & 116 & 70 & 24 & 57.5 \\
\hline 18 & 120 & 120 & 120 & 70 & 23.9 & 48.9 \\
\hline
\end{tabular}

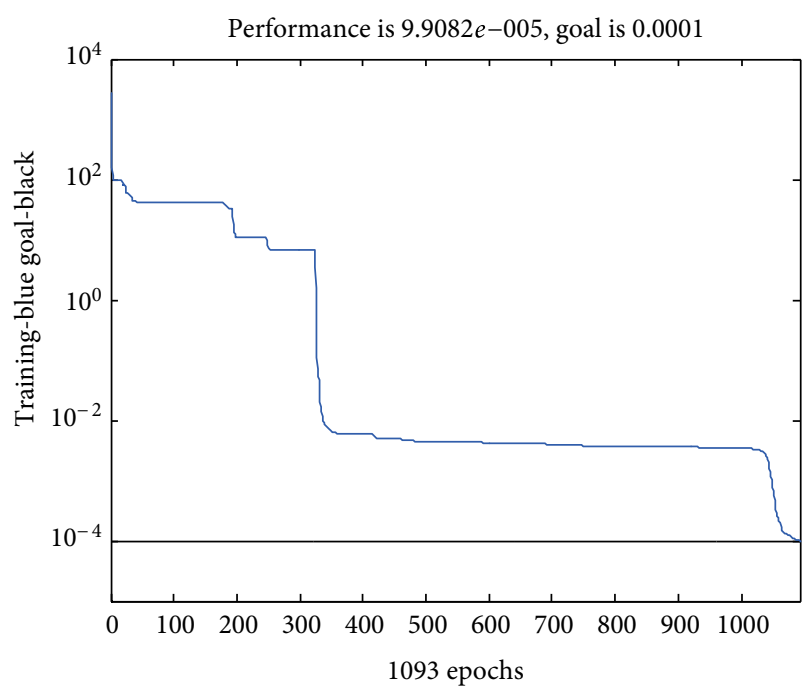

FIGURE 8: Error function values versus training times.
The polymerization time was reduced to 8.5 hours form 10 hours when the amount of initiator and emulsion of original formula were increased by $20 \%$. Every $5 \%$ increase of emulsion amount, the reductions of reaction time were different, the higher amount of emulsion, the less reduction of reaction time.

3.1.3. Influence of Molecular Regulator Amount on Polymerization Rate. The 10\%, 16\%, 20\% molecular regulator was added to reactor after the reaction 0 hour, 1 hour, 2 hour, respectively, when the amounts of initiator and emulsion of original formula were increased by $20 \%$. The reaction was terminated when the monomer conversion was $70 \%$ for avoiding the impact of sampling. The Mooney viscosities of flocculated rubber were determined. The results were shown in Figure 6.

The results show that the polymerization rate can be increased by increasing the amount of emulsion significantly. The polymerization time was reduced to 8.5 hours form 
TABLE 2: The comparison of experimental and simulation data.

\begin{tabular}{lccc}
\hline & Simulation date & Experimental data & Relative error, \% \\
\hline Combining styrene content, \% & 23.9532 & 24 & 0.195 \\
Mooney viscosity, $\mathrm{ML}_{1+4}^{100^{\circ} \mathrm{C}}$ & 57.5101 & 57.5 & 0.018 \\
Combining styrene content, \% & 23.9013 & 23.9 & 0.054 \\
Mooney viscosity, $\mathrm{ML}_{1+4}^{100^{\circ} \mathrm{C}}$ & 48.9 & 48.9 & 0 \\
\hline
\end{tabular}

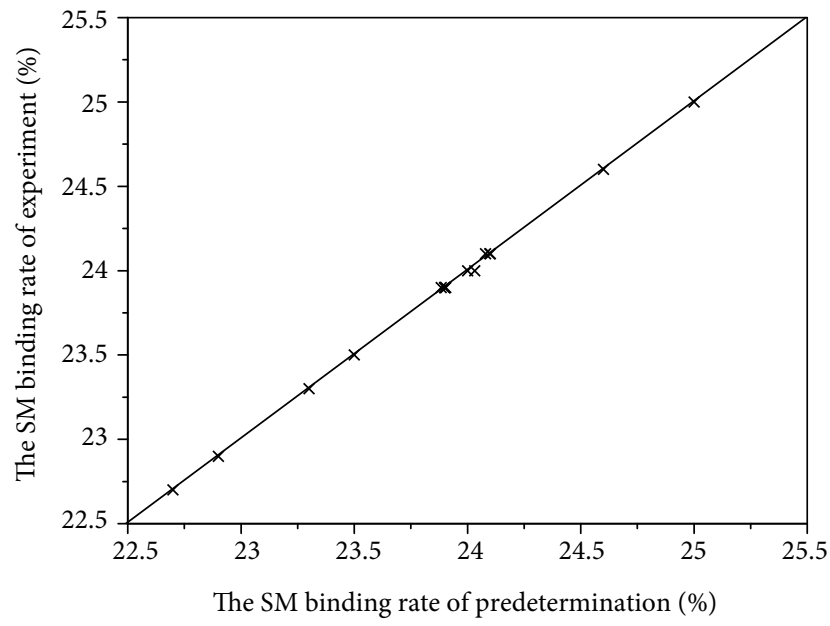

(a)

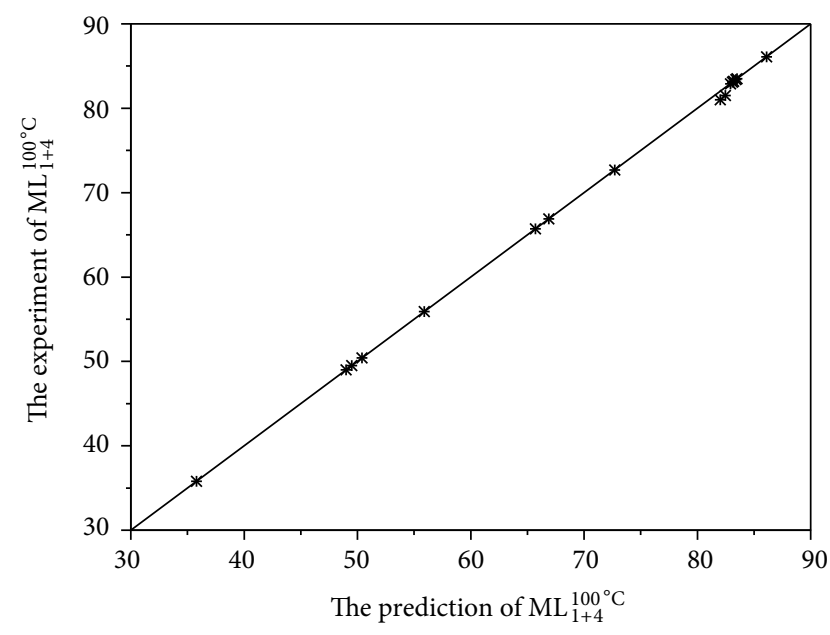

(b)

FIgURE 9: (a) The SM binding rate comparison of BP neural network simulation results and experimental values. (b) The Mooney viscosity comparison of BP neural network simulation results and experimental values.

10 hours when the amount of initiator and emulsion of original formula were increased by $20 \%$. Every $5 \%$ increase of emulsion amount, the reductions of reaction time were different, the higher amount of emulsion, the less reduction of reaction time.

The figure shows that the higher amount of molecular regulator, the lower Mooney viscosity when the initial addition amount was $100 \%$ and the polymerization times were same; the Mooney viscosity decreased firstly and increased subsequently with the delay of addition time when the additional amount were same, and it reached a nadir when the addition time was after reacting for 1-2 hours. The Mooney viscosity of rubber can meet the Chinese national standard GB8655-88 when increasing the amount of initiator to $120 \%$ original, increasing the amount of emulsion to $120 \%$ original, the initial amount of molecular regulator was $100 \%$ original, and adding 20\% more after reacting 1-2 hours.

To sum up, the polymerization rate of butadiene styrene rubber can be accelerated by $30 \%$ via increasing the initiator and emulsion amount. The Mooney viscosity of $70 \%$ conversion rate rubber can meet the Chinese national standard together with adding additional molecular regulator.

\subsection{The Foundation of BP Neural Network Model}

3.2.1. The Foundation of BP Neural Network Model. According to the product of butadiene styrene rubber, the actual situation was simulated predictively (the summary affection of formula condition to rubber was shown in Table 1). There were two hidden layer BP neural network, the first layer is linear, reflecting the influence of each condition on the product. The second layer is nonlinear, reflecting the influence of each interaction factor, and both used the sigmoid logarithmic type function model. The linear transfer function was used for the output layer. The hidden layer had 10 neurons and the output layer had 2. The BP neural network is shown in Figure 7.

On the basis of original formula and optimized formula, the input vector of BP network train were the proportion of original initiator, emulsion, molecular regulator, and the conversion rate (such as the data of 1-16 in Table 1), the target vector was the combining styrene content and Mooney viscosity of the product of butadiene styrene rubber. The network was trained by using the Levenberg-Marquardt (LM) [10] model.

3.2.2. The Simulation of BP Neural Network Model. The predictive performance was achieved after 1093 trains by Levenberg-Marquardt algorithms, $\varepsilon(W)<1 \times 10^{-4}$. The relationship of error function and training number is shown in Figure 8.

Figure 9 shows that the error of simulation result and experiment data is less than $1 \%$. They have good consistency. The correlation coefficient $R^{2}$ of experiment and predicted values is 0.985 .

The comparison between the simulation results of $17 \#$, 18\#, and experiment is shown in Table 2, and the error 
is less than $0.2 \%$. The product properties data is based on the experimental results of different formula and particular situation, so the predicted results of BP neural network are the rubber's properties data in this polymerization situation.

\section{Conclusions}

(1) The polymerization rate of butadiene styrene rubber can be accelerated by $30 \%$ via increasing the initiator and emulsion amount. The Mooney viscosity of $70 \%$ conversion rate rubber can meet the Chinese national standard together with adding additional molecular regulator.

(2) On the basis of original formula and optimized formula, the input vector of BP network train was composed of the proportion of original initiator, emulsion, molecular regulator, and the conversion rate; the target vector was composed of the combining styrene content and Mooney viscosity of the product of butadiene styrene rubber. The network was trained by using the Levenberg-Marquardt (L-M) model. The error of simulation result and experiment data is less than $1 \%$. They have good consistency. The correlation coefficient $R^{2}$ of experiment and predicted values is 0.985. So the established BP neural network model can predict the styrene binding rate and Mooney viscosity on this polymerization situation.

(3) On the basis of experiment, the established BP neural network model can predict the product properties of different formula and particular situation. It can provide reference to adjust emulsion polymerized butadiene styrene rubber formula and predict the product properties. It is also used for optimizing formula, improving raw material usage, and reducing the energy consumption.

\section{References}

[1] L. Shulai, S. Yan, Z. Liu et al., "Influence factors of increasing conversion of emulsion styrene butadiene rubber," China Synthetic Rubber Industry, vol. 29, no. 4, pp. 245-249, 2006.

[2] S. Lixin, "Kuai su gao zhuan hua lv ding ben xiang jiao ji shu kai fa qu de jie duan xing cheng guo," China Elastomerics, vol. 13, no. 5, p. 20, 2003.

[3] J. Yan-jiang, S. Ben-xian, S. Jun, C. Ming-hai, Y. Lei, and Z. Ji-gang, "The intermediates properties of SBR in emulsion polymerization by a recipe with high monomer conversion," China Elastomerics, vol. 21, no. 5, pp. 44-47, 2011.

[4] G. Martinez Delfa, A. Olivieri, and C. E. Boschetti, "Multiple response optimization of styrene-butadiene rubber emulsion polymerization," Computers and Chemical Engineering, vol. 33, no. 4, pp. 850-856, 2009.

[5] G. Padmavathi, M. G. Mandan, S. P. Mitra, and K. K. Chaudhuri, "Neural modelling of Mooney viscosity of polybutadiene rubber," Computers and Chemical Engineering, vol. 29, no. 7, pp. 1677-1685, 2005.

[6] J. Zhang, A. J. Morris, E. B. Martin, and C. Kiparissides, "Prediction of polymer quality in batch polymerisation reactors using robust neural networks," Chemical Engineering Journal, vol. 69, no. 2, pp. 135-143, 1998.

[7] G. M. Delfa and C. E. Boschetti, "Optimization of the chain transfer agent incremental addition in SBR emulsion polymerization," Journal of Applied Polymer Science, vol. 124, no. 4, pp. 3468-3477, 2012.

[8] H. Pan, Y. Chen, Y. Cai, and B.-G. Li, "Application of artificial neural networks in polymerization process," Chemical Industry and Engineering Progress, vol. 21, no. 5, pp. 314-318, 2002.

[9] L. Hao and B. Zhenxing, "Realization and applications of BP network on matlab," Modern Electronics Technique, vol. 29, no. 2, pp. 49-54, 2006.

[10] G. Jun, The Theory of Neural Network and Its Simulation, China Machine Press, Beijing, China, 2011. 

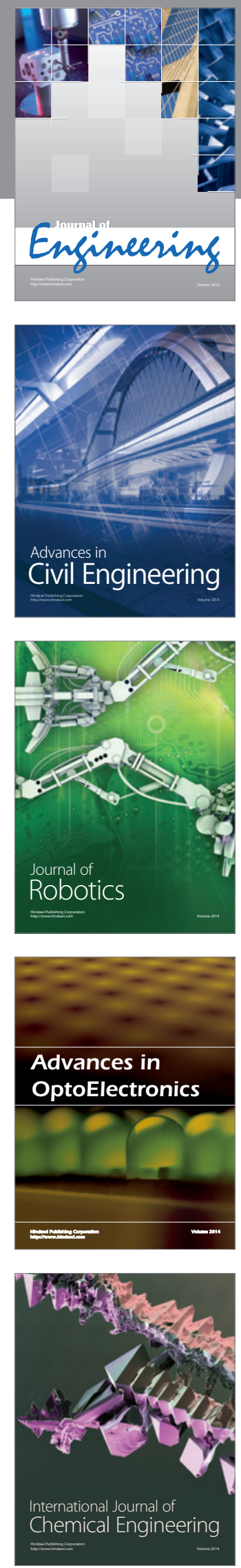

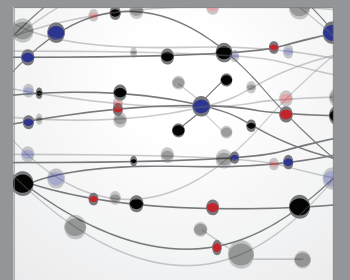

The Scientific World Journal
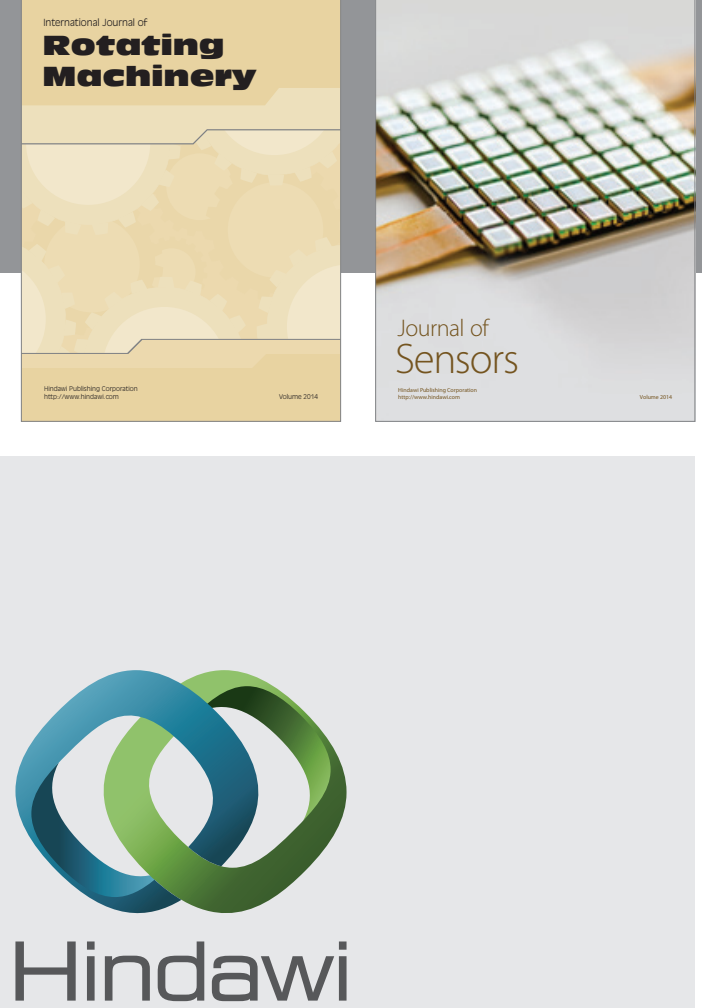

Submit your manuscripts at http://www.hindawi.com
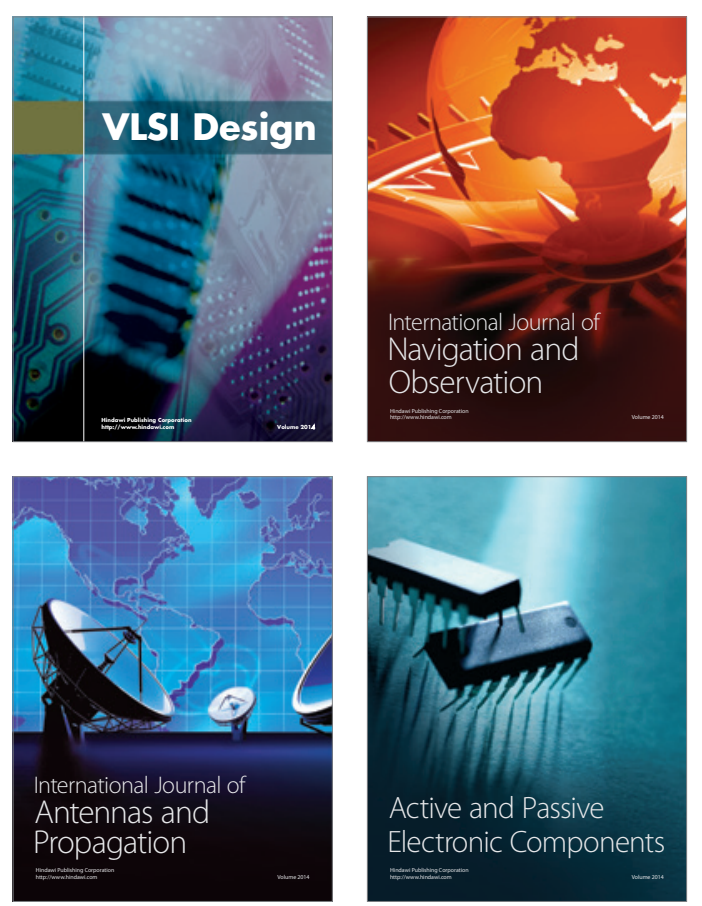
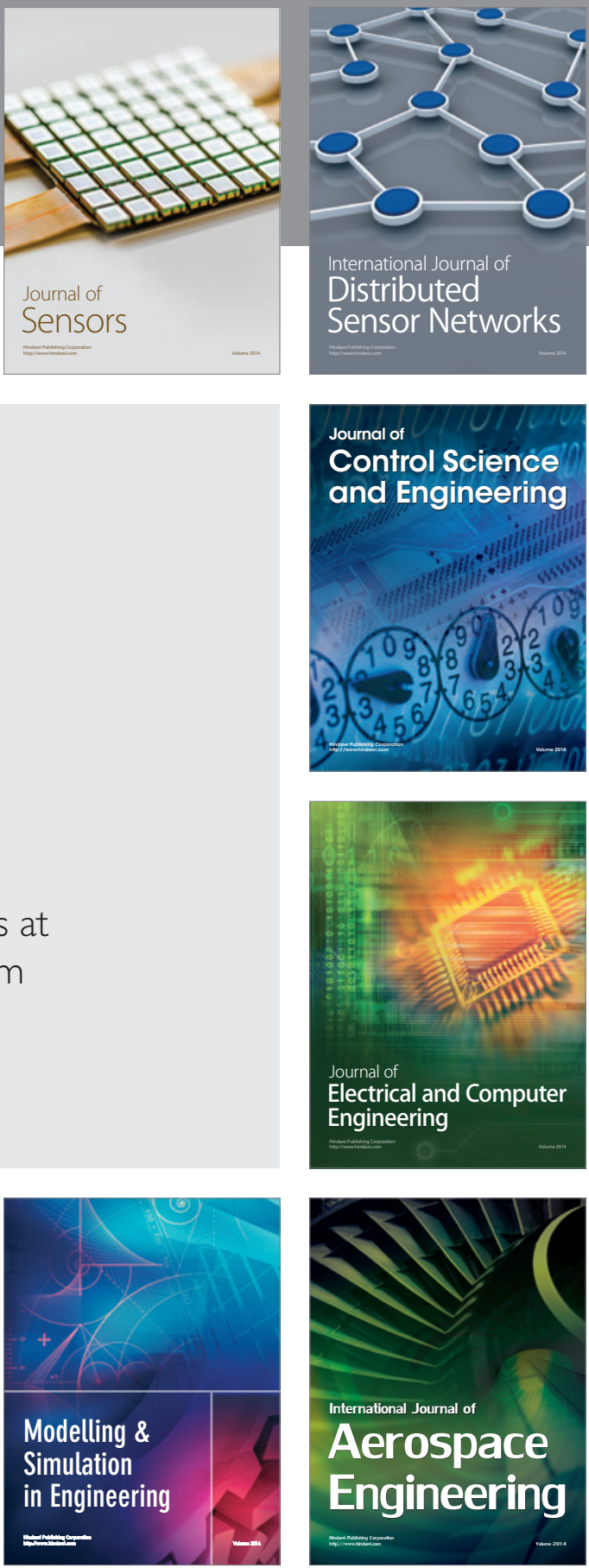

Journal of

Control Science

and Engineering
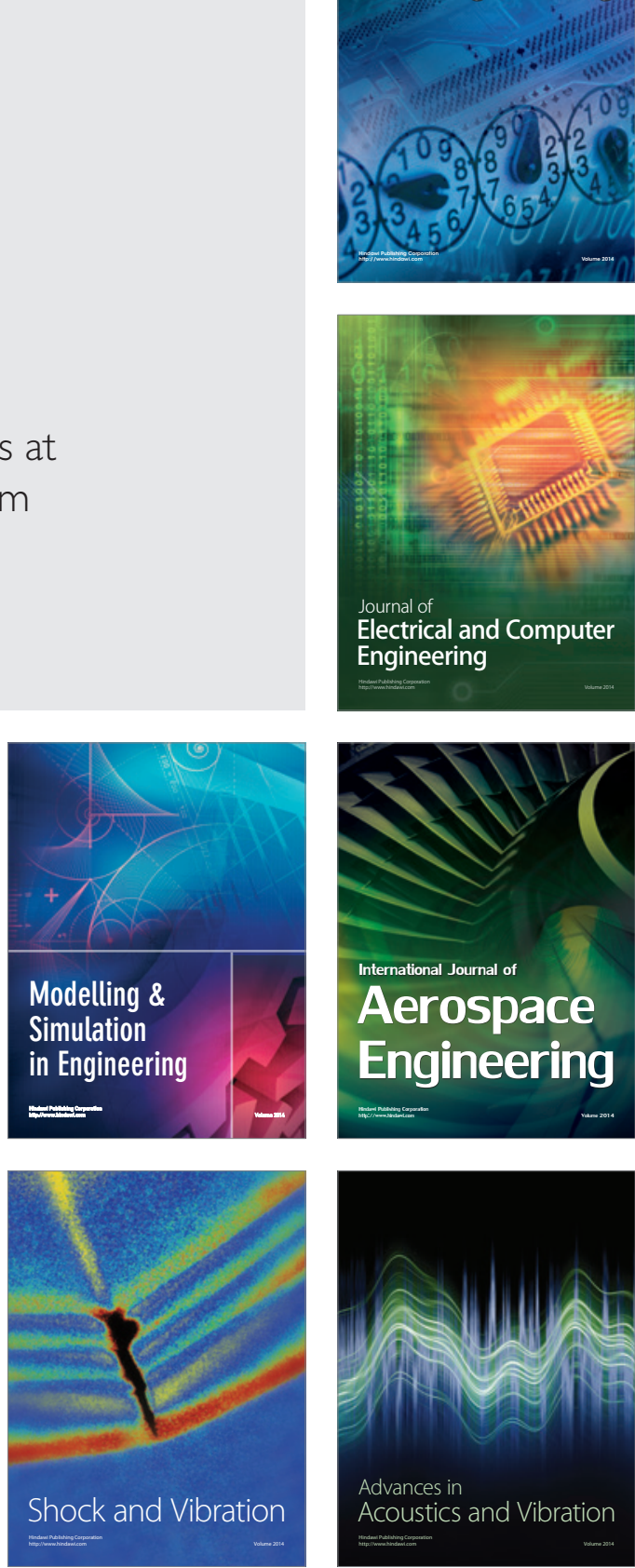